\title{
Bilirubin Nomogram: A Prediction Tool or Natural History Profile?
}

\author{
VINOD K BHUTANI \\ Stanford University School of Medicine and Lucile Packard Children's Hospital, \\ Department of Pediatrics, Division of Neonatal and Developmental Medicine, 750 Welch Ave \#315; Stanford, CA 94304. \\ bhutani@stanford.edu
}

$\mathrm{B}$ ilirubin nomograms have dual functions: $(a)$ to track an individual baby using an evidencebased predictive risk assessment tool; and, $(b)$ to inform the public health perspective on adverse outcome reduction by altering the natural course of the disease through early intervention. The original report that demonstrated the predictive value of a routine predischarge total serum bilirubin (TSB), measured as a universal screen, was simplified for immediate applicability to plot on an hour-specific bilirubin nomogram described for a population of racially diverse, healthy, term and near-term newborns during the first postnatal week in a Philadelphia hospital [1]. Subsequent studies have validated this predictive ability and the usefulness of this nomogram in several regional and multi-national populations [2]. When used with combination of TSB and transcutaneous testing, other centers have effectively promoted universal bilirubin screening $[3,4]$. The outcome measure is the likelihood (LR) of TSB $>95^{\text {th }}$ percentile for age in hours. Based on the prevalence of "disease": TSB in the high-risk zone, $>95^{\text {th }}$ percentile post-discharge for any population is (present / (present + absent)). One out of 23 infants in this study evidenced "disease". The LR may calculate a revised or posttest probability of disease: posttest odds $=$ pretest odds $\times$ LR where odds $=$ probability $\div(1-$ probability). Because LR is prevalence independent, it can be used to calculate the posttest probability at any site in the world. Alternatively, the revised probability may be obtained for any site by dividing the absent component of the present : absent ratio for the total population by the LR to calculate the revised present : absent ratio for each risk zone (legend for Table 3 in reference 1). The clinical goal for any predictive test is defined by its outcome. We selected an identification of a higher-risk population to ensure a safety margin because of known imprecision of clinical diagnostic tests. Biological risk factors, accuracy as well as precision of bilirubin, pattern of clinical practice and access to healthcare markedly influence the predictive performance of the bilirubin tests. Gestational age, race and ethnicity are also singularly important $[4$, 5]. Subsequent consensus for TSB thresholds for use phototherapy governed by prematurity, hemolysis and clinical signs of neurotoxicity [2,4] have informed clinical practices similar to those in North America and Europe. However, geography and nationality are poor surrogates for biology, race, ethnicity and prematurity and have yet to be recognized as specific risk factors. Performance of a predictive test is also largely defined, in a research setting, with meticulous attention to study design, definitions, minimization of bias, subsequent validation in diverse cohorts and an anticipation of variable implementation in actual clinical practice. In this issue of Indian Pediatrics, Pathak, et al., [6] have attempted to replicate a predictive study without addressing a precise hypothesis and have expended immense effort without studying the vulnerability (if any) for their specific population cared for at their institution. Their data could be influenced by prevailing clinical practice pattern, race or ethnicity but not geography. Assessment of proven predictive tools for local institutional quality improvement should be benchmarked to best practice models and encouraged. On the other hand, novel questions impact nations with additional clinical or public health burden for bilirubin neurotoxicity that includes sepsis, small for dates, postnatal hypoalbuminemia, unrecognized hemolysis, insufficient breast milk intake or access to healthcare. Here, the risk of neurotoxicity can be altered by natural history of the disease itself. A national endeavor to define thresholds for interventions relevant to the diverse population is an achievable newborn health priority to reduce the prevalence of infants with TSB $>95^{\text {th }}$ percentile, use of exchange transfusion or possibly prevent kernicterus in India.

Competing interests: None stated; Funding: Nil.

\section{REFERENCES}

1. Bhutani VK, Johnson LH, Sivieri EM. Predictive ability of a predischarge hour-specific serum bilirubin for subsequent significant hyperbilirubinemia in healthy term and near-term newborns. Pediatrics. 1999;103:6-14.

2. Stevenson DK, Fanaroff AA, Maisels MJ, Young BW, Wong RJ, Vreman HJ, et al. Prediction of 
EDITORIAL

hyperbilirubinemia in near-term and term infants. $\mathrm{J}$ Pediatr. 2001;108:31-9.

3. Maisels MJ, Bhutani VK, Bogen D, Newman TB, Stark AR, Watchko JF. Hyperbilirubinemia in the newborn infant $>$ or $=35$ weeks' gestation: an update with clarifications. Pediatrics. 2009;124:1193-8.

4. Bhutani VK, Stark AR, Lazzeroni LC, Poland R, Gourley GR, Kazmierczak S, et al. Initial clinical testing evaluation and risk assessment for universal screening for hyperbilirubinemia study group. Predischarge screening for severe neonatal hyperbilirubinemia identifies infants who need phototherapy. J Pediatr. 2012 Oct 5.

5. Bhutani VK. Public policy to prevent severe neonatal hyperbilirubinemia. In: Stevenson DK, Maisels MJ, Watchko JF, Eds. Care of the Jaundiced Neonate. China: McGraw-Hill Companies Inc; 2012. p 243-262.

6. Pathak U, Chawla D, Kaur S, Jain S. Bilirubin nomogram for prediction of significant hyperbilirubinemia in North Indian neonates. Indian Pediatr. 2013;50:383-9. 